\title{
Linear Weyl Gravity in de Sitter Universe
}

\author{
M.V. Takook ${ }^{1,2 *}$, M. Reza Tanhayi ${ }^{3 \dagger}$
}

October 30, 2018

\author{
${ }^{1}$ Department of Physics, Razi University, Kermanshah, Iran \\ ${ }^{2}$ Groupe de physique des particules, Université de Montréal, \\ C.P. 6128, succ. centre-ville, Montréal, Québec, Canada H3C 3J7 \\ ${ }^{3}$ Department of Physics, Islamic Azad University, Central Tehran Branch, Tehran, Iran
}

\begin{abstract}
In this paper we obtain the linear Weyl gravity in de Sitter background. Its linear form in five-dimensional ambient space notation has also been obtained. From the group theoretical point of view, we show that this conformal invariant fourth order theory in its linear form does not transform as an unitary irreducible representation of the de Sitter group.
\end{abstract}

PACS numbers: $04.62 .+\mathrm{v}, 98.80 . \mathrm{Cq}, 12.10 . \mathrm{Dm}$

\section{Introduction}

Conformal transformations and conformal techniques have been widely used in general relativity for a long time (Ref. [1] and references therein). It has been often claimed that conformal invariant field theories are renormalizable [2] and conformal gravity may be an alternative theory of gravity [3].

Since the gravitational field is long range and seems to travel with the speed of light, in the linear approximation, at least, its equations is expected to be conformally invariant. As one knows Einstein's theory of gravitation is not conformally invariant. Because of this fact and other issues arising from standard cosmology and quantum field theory [4], it seems this theory could not be considered as a comprehensive universal theory of gravitational field. Many attempts in generalizing this theory dating back to the early days of general relativity (for reviewing see [5]). The first gravitational theory which is invariant under the scale transformation was presented by Weyl, hence it is called Weyl gravity. The Conformal Weyl gravity is based on local conformal invariance of the metric of the form $g_{\mu \nu}(x) \rightarrow \Omega^{2}(x) g_{\mu \nu}(x)$, and leads to a theory with the field equation of fourth order derivative (higher-derivative theories). In a series

*e-mail: takook@razi.ac.ir

†e-mail: m_tanhayi@iauctb.ac.ir 
of papers [6], Mannheim and Kazanas explored the structure of conformal Weyl gravity as a possible alternative to the Einstein's theory. With this theory they could potentially explain two outstanding astrophysical issues namely the cosmological constant [7] and galactic rotation curve [8] problems. The related works can be found in Ref.s [9, 10, 11]. Accordingly, in this work, we study the cconformally invariant Weyl gravity in de Sitter (dS) space from the group theoretical point of view.

Gravitational field, in the linear approximation, resembles as a massless particle with spin-2 that propagates on the background space-time. According to Wigner's theorem, a linear gravitational field should transform under the unitary irreducible representation (UIR) of symmetric group of the background space-time. In this paper dS space-time has been chosen as the background. We have shown that linear Weyl gravity can not be associated with any UIR of dS group. This result may easily extended for the global scale-invariant action i.e., invariant under $g_{\mu \nu}(x) \rightarrow \Omega^{2} g_{\mu \nu}(x)$ where $\Omega$ is independent of the space-time coordinates $[12,13]$.

The organization of this paper is as follows. Section 2 is devoted to a brief review of the notations. Linear Weyl gravity equation in the intrinsic dS coordinate is calculated in this section. In section 3 we project this equation into the five-dimensional ambient space and we explore the possible relations between this field and the UIRs of the dS and conformal groups. The results are summarized and discussed in section 4. Finally, some properties of the Casimir operators and mathematical relations are presented in the appendices.

\section{Notation}

Quantum field theory in dS space has evolved as an exceedingly important subject, studied by many authors over the course of the past decade. This is due to the fact that recent astrophysical data indicate that our universe might currently be in a dS phase. The importance of dS space has been primarily ignited by the study of the inflationary model of the universe and quantum gravity. The de Sitter metric is a solution of the cosmological Einstein's equation with positive constant $\Lambda$. It is conveniently described as a hyperboloid embedded in a five-dimensional Minkowski space

$$
X_{H}=\left\{x \in \mathbb{R}^{5} ; x^{2}=\eta_{\alpha \beta} x^{\alpha} x^{\beta}=-H^{-2}=-\frac{3}{\Lambda}\right\},
$$

where $\eta_{\alpha \beta}=\operatorname{diag}(1,-1,-1,-1,-1)$ and $H$ is the Hubble parameter. The dS metric reads

$$
d s^{2}=\eta_{\alpha \beta} d x^{\alpha} d x^{\beta}=g_{\mu \nu}^{d S} d X^{\mu} d X^{\nu}
$$

where the $X^{\mu}$ are 4 space-time intrinsic coordinates of the $\mathrm{dS}$ hyperboloid. In this paper we take $\alpha, \beta, \gamma, \delta, \eta=0,1,2,3,4$ and $\lambda, \mu, \nu, \rho, \sigma, \tau=0,1,2,3$. Any geometrical object in this space can be written in terms of the four local coordinates $X^{\mu}$ (intrinsic) or in terms of the five global coordinates $x^{\alpha}$ (ambient space).

\section{I) Fourth order Weyl equation:}

Consider a space-time $\left(\mathcal{M}, g_{\mu \nu}\right)$, where $\mathcal{M}$ is an $n$-dimensional manifold with metric $g_{\mu \nu}$ of any signature. The following transformation

$$
g_{\mu \nu} \rightarrow g_{\mu \nu}^{\prime}=\Omega^{2}(x) g_{\mu \nu}
$$


where $\Omega(x)$ is a nonvanishing, regular function, is called a Weyl or scale transformation and it leaves the light cones unchanged [12]. The global scale-invariant action in the metric signature $(-,+,+,+)$ is $[12]$

$$
I_{g}=-\frac{1}{4} \int d^{4} x \sqrt{-g}\left(a C_{\mu \nu \lambda \rho} C^{\mu \nu \lambda \rho}+b R^{2}\right),
$$

where $a, b$ are two dimensionless constant parameters, $g=\operatorname{det}\left(g_{\mu \nu}\right)$ and $C_{\mu \nu \lambda \rho}$ is the conformal Weyl tensor. The action which is invariant under the local conformal transformation (2.1) is described by $\left(a=4 \alpha_{g}, b=0\right)[13]$

$$
I_{W}=-2 \alpha_{g} \int d^{4} x \sqrt{-g}\left(R_{\mu \nu} R^{\mu \nu}-\frac{1}{3} R^{2}\right)+(\text { surface term })
$$

where $\alpha_{g}$ is a necessarily dimensionless gravitational coupling constant. Note that in four dimensions because of Gauss-Bonnet theorem the contribution of $R_{\mu \nu \lambda \rho} R^{\mu \nu \lambda \rho}$ in to the action can be written in terms of $R^{2}$ and $R_{\mu \nu} R^{\mu \nu}$. The total action is $I \equiv I_{W}+I_{M}$, where $I_{M}$ is the conformal matter action (for example see [10]). Functional variation of the total action with respect to the matter fields yields the equations of motion while its functional variation with respect to the metric yields the Weyl field equation. So the Weyl field equation becomes as follows

$$
W_{\mu \nu} \equiv W_{\mu \nu}^{(2)}-\frac{1}{3} W_{\mu \nu}^{(1)}=\frac{1}{4 \alpha_{g}} T_{\mu \nu}
$$

where $T_{\mu \nu} \equiv \frac{\delta I_{M}}{\delta g^{\mu \nu}}$ is the energy-momentum tensor. It is given by Eq. (7) in Ref. [10], and ${ }^{1}$

$$
\begin{gathered}
W_{\mu \nu}^{(1)}=-2 g_{\mu \nu} \square R+2 \nabla_{\mu} \nabla_{\nu} R-2 R R_{\mu \nu}+\frac{1}{2} g_{\mu \nu} R^{2}, \\
W_{\mu \nu}^{(2)}=-\frac{1}{2} g_{\mu \nu} \square R-\square R_{\mu \nu}+\nabla_{\lambda} \nabla_{\nu} R_{\mu}^{\lambda}+\nabla_{\lambda} \nabla_{\mu} R_{\nu}^{\lambda}-2 R_{\mu}^{\lambda} R_{\nu \lambda}+\frac{1}{2} g_{\mu \nu} R_{\rho \lambda} R^{\rho \lambda} .
\end{gathered}
$$

It can be shown that $W_{\mu \nu}$ is covariantly conserved and traceless. So this theory leads a fourth order theory of gravity, it is sometimes known as $R^{2}$-gravity theory.

\section{II) Linear field equation in de Sitter space:}

Now we want to obtain the linear approximation of the free field equation in dS background. In the background field method, $g_{\mu \nu}=g_{\mu \nu}^{B G}+h_{\mu \nu}$, we suppose $g_{\mu \nu}^{B G}=g_{\mu \nu}^{d S}$, so we have

$$
g_{\mu \nu}=g_{\mu \nu}^{d S}+h_{\mu \nu}, \quad g_{\mu \nu}^{d S} \equiv \tilde{g}_{\mu \nu}, \quad g^{\mu \nu}=\tilde{g}^{\mu \nu}-h^{\mu \nu}+\mathcal{O}\left(h^{2}\right)
$$

Taking the first variation of $W_{\mu \nu}$ under the small metric variation (2.5) and preserving only linear terms of $h_{\mu \nu}$ results in (appendix B)

$$
W_{\mu \nu}^{(1)-L}=12 H^{2} \square h_{\mu \nu}+72 H^{4} h_{\mu \nu}+2 \tilde{g}_{\mu \nu}\left(\square^{2} h-\square \nabla \cdot \nabla \cdot h+3 H^{2} \nabla \cdot \nabla \cdot h-9 H^{4} h\right)
$$

\footnotetext{
${ }^{1}$ In this paper we follow the sign convention of Ref. [12] namely

$$
\begin{gathered}
R_{b c d}^{a}=\partial_{c} \Gamma_{b d}^{a}+\Gamma_{e c}^{a} \Gamma_{b d}^{e}-c \leftrightarrow d, \\
R_{b d}=+R_{b a d}^{a}, \text { and } R=+R_{a}^{a} .
\end{gathered}
$$
}




$$
\begin{gathered}
+2\left(\nabla_{\mu} \nabla_{\nu} \nabla \cdot \nabla \cdot h-\nabla_{\mu} \nabla_{\nu} \square h-6 H^{2} \nabla_{\lambda} \nabla_{\mu} h_{\nu}^{\lambda}-6 H^{2} \nabla_{\lambda} \nabla_{\nu} h_{\mu}^{\lambda}+3 H^{2} \nabla_{\mu} \nabla_{\nu} h\right), \\
W_{\mu \nu}^{(2)-L}=36 H^{4} h_{\mu \nu}+9 H^{2} \square h_{\mu \nu}+\frac{1}{2} \tilde{g}_{\mu \nu}\left(\square^{2} h-\square \nabla \cdot \nabla \cdot h-3 H^{2} \square h+6 H^{2} \nabla \cdot \nabla \cdot h-18 H^{4} h\right) \\
+6 H^{2} \nabla_{\mu} \nabla_{\nu} h+\frac{1}{2} \square\left(\square h_{\mu \nu}+\nabla_{\mu} \nabla_{\nu} h-\nabla_{\lambda} \nabla_{\mu} h_{\nu}^{\lambda}-\nabla_{\lambda} \nabla_{\nu} h_{\mu}^{\lambda}\right)-9 H^{2}\left(\nabla_{\lambda} \nabla_{\mu} h_{\nu}^{\lambda}+\nabla_{\lambda} \nabla_{\nu} h_{\mu}^{\lambda}\right) \\
-\frac{1}{2} \nabla_{\lambda} \nabla_{\mu}\left(\square h_{\nu}^{\lambda}+\nabla^{\lambda} \nabla_{\mu} h-\nabla_{\rho} \nabla_{\mu} h^{\lambda \rho}-\nabla_{\rho} \nabla^{\lambda} h_{\nu}^{\rho}\right) \\
-\frac{1}{2} \nabla_{\lambda} \nabla_{\nu}\left(\square h_{\mu}^{\lambda}+\nabla^{\lambda} \nabla_{\nu} h-\nabla_{\rho} \nabla_{\nu} h^{\lambda \rho}-\nabla_{\rho} \nabla^{\lambda} h_{\mu}^{\rho}\right) .
\end{gathered}
$$

Thus the linear free field Weyl equation (2.4) can be written in de Sitter background as follows

$$
W_{\mu \nu}^{L} \equiv W_{\mu \nu}^{(2)-L}-\frac{1}{3} W_{\mu \nu}^{(1)-L}=0
$$

or

$$
\begin{gathered}
2 W_{\mu \nu}^{L}=\square^{2} h_{\mu \nu}-6 H^{2} \square h_{\mu \nu}+8 H^{4} h_{\mu \nu}-H^{2} \nabla_{\mu} \nabla \cdot h_{\nu}-H^{2} \nabla_{\nu} \nabla \cdot h_{\mu}+\frac{2}{3} \nabla_{\mu} \nabla_{\nu} \nabla \cdot \nabla \cdot h \\
+\tilde{g}_{\mu \nu}\left(\frac{1}{3} \square \nabla \cdot \nabla \cdot h-\frac{1}{3} \square^{2} h+2 H^{2} \nabla \cdot \nabla \cdot h+H^{2} \square h-2 H^{4} h\right)+\frac{1}{3} \nabla_{\mu} \nabla_{\nu} \square h \\
+2 H^{2} \nabla_{\mu} \nabla_{\nu} h+\frac{1}{3} \nabla_{\mu} \nabla_{\nu} \square h-\nabla_{\mu} \square \nabla \cdot h_{\nu}-\nabla_{\nu} \square \nabla \cdot h_{\mu}=0 .
\end{gathered}
$$

where the box operator $\square=\tilde{g}_{\mu \nu} \nabla^{\mu} \nabla^{\nu}$, is the Laplace-Beltrami operator in the dS space. In the process of getting above relation, we used (appendix B)

$$
\begin{gathered}
\nabla_{\lambda} \nabla_{\mu} h_{\nu}^{\lambda}=\nabla_{\mu} \nabla \cdot h_{\nu}+4 H^{2} h_{\mu \nu}-H^{2} \tilde{g}_{\mu \nu} h, \\
\nabla_{\lambda} \square h_{\rho}^{\lambda}=\square \nabla \cdot h_{\rho}+5 H^{2} \nabla \cdot h_{\rho}-2 H^{2} \nabla_{\rho} h, \\
\nabla_{\lambda} \nabla_{\nu} \square h_{\mu}^{\lambda}=\nabla_{\nu} \square \nabla \cdot h_{\mu}+5 H^{2} \nabla_{\nu} \nabla \cdot h_{\mu}-2 H^{2} \nabla_{\nu} \nabla_{\mu} h+4 H^{2} \square h_{\mu \nu}-H^{2} \tilde{g}_{\mu \nu} \square h .
\end{gathered}
$$

The flat limit of the Eq. (2.9) can be easily obtain by taking the Hubble constant equal to zero as follows

$$
\square^{2} h_{\mu \nu}+\frac{2}{3} \partial_{\mu} \partial_{\nu} \partial \cdot \partial \cdot h+\eta_{\mu \nu}\left(\frac{1}{3} \square \partial \cdot \partial \cdot h-\frac{1}{3} \square^{2} h\right)+\frac{1}{3} \partial_{\mu} \partial_{\nu} \square h-\partial_{\mu} \square \partial \cdot h_{\nu}-\partial_{\nu} \square \partial \cdot h_{\mu}=0,
$$

in the flat limit $\square=\partial_{\mu} \partial^{\mu}$ is D'Alembertian operator. Now let us consider the Eq. (2.9), by imposing the traceless and divergenceless conditions on $h_{\mu \nu}$ i.e.,

$$
h_{\mu}^{\mu}=h=0, \quad \nabla_{\mu} h_{\nu}^{\mu}=\nabla \cdot h_{\nu}=0,
$$

which are important in considering the UIR of dS group, we get the following field equation

$$
\square^{2} h_{\mu \nu}-6 H^{2} \square h_{\mu \nu}+8 H^{4} h_{\mu \nu}=0 .
$$

In order to explore the possible relation between this field equation and the UIR of the de Sitter group in the next section we translate this equation into the ambient space notation, since in this notation the Casimir operators of the de Sitter group, SO(1,4), take a simple form and also the UIRs of the de Sitter group are easy to consider (appendix A). 


\section{Linear field equation in ambient space notation}

In order to clarify the relation between field equation and the representation of the dS group, we have adopted the tensor field $\mathcal{K}_{\alpha \beta}(x)$ in ambient space notation. In this notation, the relationship with UIRs of the dS group becomes straightforward because the Casimir operators are easily identified with the field equation [14]. The transverse tensor field $\mathcal{K}_{\alpha \beta}(x)$ is locally determined by the "intrinsic" field $h_{\mu \nu}(X)$ through

$$
h_{\mu \nu}(X)=\frac{\partial x^{\alpha}}{\partial X^{\mu}} \frac{\partial x^{\beta}}{\partial X^{\nu}} \mathcal{K}_{\alpha \beta}(x(X)) .
$$

The symmetric tensor field $\mathcal{K}_{\alpha \beta}(x)$ is defined in dS space and will be viewed here as a homogeneous function, with some arbitrarily chosen degree $\sigma$, in the $\mathbb{R}^{5}$-variables $x^{\alpha}[15]$

$$
x^{\alpha} \frac{\partial}{\partial x^{\alpha}} \mathcal{K}_{\beta \gamma}(x)=x \cdot \partial \mathcal{K}_{\beta \gamma}(x)=\sigma \mathcal{K}_{\beta \gamma}(x) .
$$

It also satisfies the transversality condition [15]

$$
x \cdot \mathcal{K}(x)=0 \text {, i.e. } x^{\alpha} \mathcal{K}_{\alpha \beta}(x)=0 \text {, and } x^{\beta} \mathcal{K}_{\alpha \beta}(x)=0 \text {. }
$$

To express tensor field in terms of the ambient space coordinates, transverse projection is defined

$$
(\operatorname{Trpr} \mathcal{K})_{\alpha_{1} \cdots \alpha_{l}} \equiv \theta_{\alpha_{1}}^{\beta_{1}} \cdots \theta_{\alpha_{l}}^{\beta_{l}} \mathcal{K}_{\beta_{1} \cdots \beta_{l}}
$$

The transverse projection guarantees the transversality in each index. Therefore the covariant

derivative of a tensor field, $T_{\alpha_{1} \ldots \alpha_{n}}$, in the ambient space notation becomes

$$
\operatorname{Trpr} \bar{\partial}_{\beta} \mathcal{K}_{\alpha_{1} \ldots . \alpha_{n}} \equiv \nabla_{\beta} T_{\alpha_{1} \ldots \alpha_{n}} \equiv \bar{\partial}_{\beta} T_{\alpha_{1} \ldots \alpha_{n}}-H^{2} \sum_{i=1}^{n} x_{\alpha_{i}} T_{\alpha_{1} . . \alpha_{i-1} \beta \alpha_{i+1} . . \alpha_{n}} .
$$

In the above relations $\bar{\partial}_{\alpha}$ is the tangential (or transverse) derivative in $\mathrm{dS}$ space,

$$
\bar{\partial}_{\alpha}=\theta_{\alpha \beta} \partial^{\beta}=\partial_{\alpha}+H^{2} x_{\alpha} x \cdot \partial, \quad x \cdot \bar{\partial}=0,
$$

and $\theta_{\alpha \beta}$ is the transverse projector defined by $\theta_{\alpha \beta}=\eta_{\alpha \beta}+H^{2} x_{\alpha} x_{\beta}$. Applying this procedure to a transverse second rank tensor field, leads to

$$
\mathcal{T}_{\beta \gamma \eta} \equiv \operatorname{Trpr} \bar{\partial}_{\beta} \mathcal{K}_{\gamma \eta}=\bar{\partial}_{\beta} \mathcal{K}_{\gamma \eta}-H^{2} x_{\gamma} \mathcal{K}_{\beta \eta}-H^{2} x_{\eta} \mathcal{K}_{\gamma \beta},
$$

where $\mathcal{T}_{\beta \gamma \eta}$ is now a transverse tensor field of rank 3. For transverse tensor fields of rank 3,4 and 5 , we can respectively write

$$
\begin{gathered}
\mathcal{M}_{\alpha \beta \gamma \eta} \equiv \operatorname{Trpr} \bar{\partial}_{\alpha} \mathcal{T}_{\beta \gamma \eta}=\bar{\partial}_{\alpha} \mathcal{T}_{\beta \gamma \eta}-H^{2} x_{\beta} \mathcal{T}_{\alpha \gamma \eta}-H^{2} x_{\gamma} \mathcal{T}_{\beta \alpha \eta}-H^{2} x_{\eta} \mathcal{T}_{\beta \gamma \alpha} \\
\mathcal{N}_{\delta \alpha \beta \gamma \eta} \equiv \operatorname{Trpr} \bar{\partial}_{\delta} \mathcal{M}_{\alpha \beta \gamma \eta}=\bar{\partial}_{\delta} \mathcal{M}_{\alpha \beta \gamma \eta}-H^{2} x_{\alpha} \mathcal{M}_{\delta \beta \gamma \eta}-H^{2} x_{\beta} \mathcal{M}_{\alpha \delta \gamma \eta}-H^{2} x_{\gamma} \mathcal{M}_{\alpha \beta \delta \eta}-H^{2} x_{\eta} \mathcal{M}_{\alpha \beta \gamma \delta} \\
\mathcal{P}_{\epsilon \delta \alpha \beta \gamma \eta} \equiv \operatorname{Trpr} \bar{\partial}_{\epsilon} \mathcal{N}_{\delta \alpha \beta \gamma \eta}=\bar{\partial}_{\epsilon} \mathcal{N}_{\delta \alpha \beta \gamma \eta}-H^{2} x_{\delta} \mathcal{N}_{\epsilon \alpha \beta \gamma \eta}-H^{2} x_{\alpha} \mathcal{N}_{\delta \epsilon \beta \gamma \eta}-H^{2} x_{\beta} \mathcal{N}_{\delta \alpha \epsilon \gamma \eta}-H^{2} x_{\gamma} \mathcal{N}_{\delta \alpha \beta \epsilon \eta} \\
-H^{2} x_{\eta} \mathcal{N}_{\delta \alpha \beta \gamma \epsilon} .
\end{gathered}
$$


For example replacing (4.6) in to (4.7) results in

$$
\begin{gathered}
\mathcal{M}_{\alpha \beta \gamma \eta}=\operatorname{Trpr} \bar{\partial}_{\alpha} \operatorname{Trpr} \bar{\partial}_{\beta} \mathcal{K}_{\gamma \eta}=\bar{\partial}_{\alpha}\left(\bar{\partial}_{\beta} \mathcal{K}_{\gamma \eta}-2 H^{2} x_{(\gamma} \mathcal{K}_{\eta) \beta}\right)-H^{2} x_{\beta}\left(\bar{\partial}_{\alpha} \mathcal{K}_{\gamma \eta}-2 H^{2} x_{(\gamma} \mathcal{K}_{\eta) \alpha}\right) \\
-H^{2} x_{\gamma}\left(\bar{\partial}_{\beta} \mathcal{K}_{\alpha \eta}-2 H^{2} x_{(\alpha} \mathcal{K}_{\eta) \beta}\right)-H^{2} x_{\eta}\left(\bar{\partial}_{\beta} \mathcal{K}_{\alpha \gamma}-2 H^{2} x_{(\gamma} \mathcal{K}_{\alpha) \beta}\right)
\end{gathered}
$$

By using the following relations [16]

$$
\begin{gathered}
g_{\mu \nu}^{d S}=\frac{\partial x^{\alpha}}{\partial X^{\mu}} \frac{\partial x^{\beta}}{\partial X^{\nu}} \theta_{\alpha \beta} \\
\nabla_{\mu} \cdots \nabla_{\rho} h_{\lambda_{1} \cdots \lambda_{l}}=\frac{\partial x^{\alpha}}{\partial X^{\mu}} \cdots \frac{\partial x^{\gamma}}{\partial X^{\rho}} \frac{\partial x^{\eta_{1}}}{\partial X^{\lambda_{1}}} \cdots \frac{\partial x^{\eta_{l}}}{\partial X^{\lambda_{l}}} \operatorname{Trpr} \bar{\partial}_{\alpha} \cdots \operatorname{Trpr} \bar{\partial}_{\gamma} \mathcal{K}_{\eta_{1} \cdots \eta_{l}},
\end{gathered}
$$

we obtain

$$
\begin{gathered}
\nabla_{\mu} \nabla \cdot h_{\nu}=\frac{\partial x^{\alpha}}{\partial X^{\mu}} \frac{\partial x^{\eta}}{\partial X^{\nu}} \mathcal{M}_{\alpha}{ }_{\beta}{ }_{\beta \eta}, \\
\square h_{\mu \nu}=\frac{\partial x^{\gamma}}{\partial X^{\mu}} \frac{\partial x^{\eta}}{\partial X^{\nu}} \mathcal{M}^{\alpha}{ }_{\alpha \gamma \eta}, \\
\square^{2} h_{\mu \nu}=\frac{\partial x^{\gamma}}{\partial X^{\mu}} \frac{\partial x^{\eta}}{\partial X^{\nu}} \mathcal{P}_{\delta}^{\delta}{ }_{\alpha \gamma \eta}{ }_{\alpha \gamma},
\end{gathered}
$$

where $\mathcal{M}^{\alpha}{ }_{\alpha \gamma \eta}, \mathcal{M}_{\alpha}{ }^{\beta}{ }_{\beta \eta}$ and $\mathcal{P}^{\delta}{ }_{\delta \alpha \gamma \eta}^{\alpha}$ are easily calculated from (3.7) and (3.9) by contraction of the indices as follows

$$
\begin{gathered}
\mathcal{M}_{\alpha \gamma \eta}^{\alpha}=\left(\bar{\partial}^{2}-2 H^{2}\right) \mathcal{K}_{\gamma \eta}-4 H^{2} x_{(\gamma} \bar{\partial} \cdot \mathcal{K}_{\eta)}, \\
\mathcal{M}_{\alpha}{ }^{\beta}{ }_{\beta \eta}=\bar{\partial}_{\alpha} \bar{\partial} \cdot \mathcal{K}_{\eta}-H^{2} x_{\eta} \bar{\partial} \cdot \mathcal{K}_{\alpha} \\
\mathcal{P}^{\delta}{ }_{\delta}{ }^{\alpha}{ }_{\alpha \gamma \eta}=\left(\bar{\partial}^{4}-4 H^{2} \bar{\partial}^{2}+4 H^{4}\right) \mathcal{K}_{\gamma \eta}-4 H^{2} \bar{\partial}^{2} x_{(\gamma} \bar{\partial} \cdot \mathcal{K}_{\eta)}+32 H^{4} x_{(\gamma} \bar{\partial} \cdot \mathcal{K}_{\eta)} \\
-4 H^{2} x_{(\gamma} \bar{\partial}^{2} \bar{\partial} \cdot \mathcal{K}_{\eta)}+8 H^{4} x_{\gamma} x_{\eta} \bar{\partial} \cdot \bar{\partial} \cdot \mathcal{K},
\end{gathered}
$$

where $2 T_{(\alpha \beta)}=T_{\alpha \beta}+T_{\beta \alpha}$. These formalism enable one to project all equations from intrinsic coordinates into the ambient space notation. So the linear Weyl equation in the ambient space notation can be written as follows

$$
\begin{gathered}
\mathcal{P}^{\delta}{ }_{\delta \gamma}{ }^{\gamma}{ }_{\alpha \beta}-6 H^{2} \mathcal{M}^{\delta}{ }_{\delta \alpha \beta}+8 H^{4} \mathcal{K}_{\alpha \beta}-H^{2} \mathcal{M}_{\alpha}{ }^{\gamma}{ }_{\gamma \beta}-H^{2} \mathcal{M}_{\beta}{ }^{\gamma}{ }_{\gamma \alpha}+\frac{2}{3} \mathcal{P}_{\alpha \beta \gamma \delta}{ }^{\gamma \delta} \\
+\theta_{\alpha \beta}\left(\frac{1}{3} \mathcal{P}_{\delta}{ }^{\delta \gamma \eta}{ }_{\gamma \eta}+2 H^{2} \mathcal{M}^{\gamma \eta}{ }_{\gamma \eta}\right)-\mathcal{P}_{\alpha}{ }^{\delta}{ }_{\delta}{ }^{\gamma}{ }_{\gamma \beta}-\mathcal{P}_{\beta}{ }^{\delta}{ }_{\delta}{ }^{\gamma}{ }_{\gamma \alpha}=0 .
\end{gathered}
$$

Note that the condition $\mathcal{K}^{\prime}=\mathcal{K}_{\alpha}^{\alpha}=0$ has been imposed. After some tedious but straightforward algebra, we find that:

$$
\begin{gathered}
\left(\bar{\partial}^{4}-10 H^{2} \bar{\partial}^{2}+24 H^{4}\right) \mathcal{K}_{\alpha \beta}-6 H^{2} x_{(\alpha} \bar{\partial}^{2} \bar{\partial} \cdot \mathcal{K}_{\beta)}+40 H^{4} x_{(\alpha} \bar{\partial} \cdot \mathcal{K}_{\beta)}-8 H^{2} \bar{\partial}_{(\alpha} \bar{\partial} \cdot \mathcal{K}_{\beta)} \\
+4 H^{4} x_{\alpha} x_{\beta} \bar{\partial} \cdot \bar{\partial} \cdot \mathcal{K}+\theta_{\alpha \beta}\left(\frac{1}{3} \bar{\partial}^{2} \bar{\partial} \cdot \bar{\partial} \cdot \mathcal{K}+6 H^{2} \bar{\partial} \cdot \bar{\partial} \cdot \mathcal{K}\right)+\frac{2}{3} \bar{\partial}_{\alpha} \bar{\partial}_{\beta} \bar{\partial} \cdot \bar{\partial} \cdot \mathcal{K} \\
+\frac{4}{3} H^{2} x_{\beta} \bar{\partial}_{\alpha} \bar{\partial} \cdot \bar{\partial} \cdot \mathcal{K}+2 H^{2} x_{\alpha} \bar{\partial}_{\beta} \bar{\partial} \cdot \bar{\partial} \cdot \mathcal{K}-2 \bar{\partial}_{(\alpha} \bar{\partial}^{2} \bar{\partial} \cdot \mathcal{K}_{\beta)}=0
\end{gathered}
$$


Useful identity in deriving this equation is

$$
x_{\alpha} \bar{\partial}^{2}=\bar{\partial}^{2} x_{\alpha}-4 H^{2} x_{\alpha}-2 \bar{\partial}_{\alpha} .
$$

By imposing the divergencelessness on $\mathcal{K}$, namely $\bar{\partial} . \mathcal{K}=0$, Eq. (3.16) reduces to

$$
\left(\bar{\partial}^{4}-10 H^{2} \bar{\partial}^{2}+24 H^{4}\right) \mathcal{K}_{\alpha \beta}=0 .
$$

This equation can be written in terms of the second order Casimir operator of the dS group as

$$
\left(Q_{0}^{2}+10 Q_{0}+24\right) \mathcal{K}_{\alpha \beta}=0, \text { or }\left(Q_{0}+4\right)\left(Q_{0}+6\right) \mathcal{K}_{\alpha \beta}=0,
$$

where $Q_{0}\left(=-H^{-2} \bar{\partial}^{2}\right)$ is the second order Casimir operator of the dS group (appendix A). On the other hand it has been proved that a symmetric tensor field of rank-2 will transform as an UIR of dS group if it satisfies the following equation [16]

$$
Q_{0}\left(Q_{0}-2\right) \mathcal{K}_{\alpha \beta}=0
$$

Clearly the Eq. (3.19) is not compatible with that in (3.18) of the linear Weyl gravity in dS space. The direct result is that the linear Weyl gravity in $\mathrm{dS}$ space (Eq. (3.18)) does not transform as an unitary irreducible representation of the dS group.

\section{Conclusion and outlook}

Conformal symmetry is indeed one of the most important measures of assessment of massless field in quantum field theory. If the graviton does exist and propagates on the light cone due to its long range effect, it should have zero mass. The light cone propagation immediately imposes the conformal invariance on the graviton field equations. In other words gravitational field should transform under the UIR of conformal group.

Although Weyl gravity is conformally invariant, it was shown that its linear form in dS space does not lead to any UIR of the dS group. So Weyl gravitational equation is not suitable to describe quantum gravitational field in the linear approximation in the dS space. Therefore, if a highly probable linear conformal quantum gravity exists, it could not be represented by the Weyl gravity. Other authors have concluded the same through different methods [2, 17].

On the other hand Barut and Böhm [18] have shown that the value of the conformal Casimir operator for the UIR must be equal to 9. By using this fact, Bineger et al. [19] proved that only mixed symmetry tensor field of rank-3 can be a physical representation of the conformal group. In the previous paper [20], we extended this result to dS space which is in agreement with the conclusion of Fronsdal et al. in anti-de Sitter space [21]. In other words if we want the linear gravitational field equations to be conformally invariant and the fields to transform according to the UIRs of their symmetry group as well as conformal group, we should write the equations with a mixed symmetry rank-3 tensor field which leads to a theory like $R^{3}$-gravity theory [26].

Acknowledgments: M.V.T. would like to thank M.B. Paranjape and S. Rouhani for their helpful discussions. We also would like to thank T. Ramezani, H. Ardahali and E. Ariamand for their interest in this work. 


\section{A de Sitter Casimir operators}

In this appendix some properties of the Casimir operators and their relation with the box operator, $\square=\tilde{g}_{\mu \nu} \nabla^{\mu} \nabla^{\nu}$, is presented. We also discuss the solution of the field equation.

There are two Casimir operators

$$
\begin{gathered}
Q^{(1)}=-\frac{1}{2} L^{\alpha \beta} L_{\alpha \beta}=-\frac{1}{2}\left(M^{\alpha \beta}+S^{\alpha \beta}\right)\left(M_{\alpha \beta}+S_{\alpha \beta}\right), \\
Q^{(2)}=-W_{\alpha} W^{\alpha}, \quad W_{\alpha}=-\frac{1}{8} \epsilon_{\alpha \beta \gamma \sigma \eta} L^{\beta \gamma} L^{\sigma \eta}
\end{gathered}
$$

where $M_{\alpha \beta}=-i\left(x_{\alpha} \partial_{\beta}-x_{\beta} \partial_{\alpha}\right)=-i\left(x_{\alpha} \bar{\partial}_{\beta}-x_{\beta} \bar{\partial}_{\alpha}\right)$ and the symbol $\epsilon_{\alpha \beta \gamma \sigma \eta}$ holds for the usual antisymmetric tensor. The action of 'spin' generator $S_{\alpha \beta}$ is defined by [14]

$$
S_{\alpha \beta} \mathcal{K}_{\gamma \delta}=-i\left(\eta_{\alpha \gamma} \mathcal{K}_{\beta \delta}-\eta_{\beta \gamma} \mathcal{K}_{\alpha \delta}+\eta_{\alpha \delta} \mathcal{K}_{\beta \gamma}-\eta_{\beta \delta} \mathcal{K}_{\alpha \gamma}\right)
$$

Following Dixmier [22], we get a classification scheme using a pair $(p, q)$ of parameters involved in the following possible spectral values of the Casimir operators:

$$
Q_{p}^{(1)}=(-p(p+1)-(q+1)(q-2)) I_{d}, \quad Q_{p}^{(2)}=(-p(p+1) q(q-1)) I_{d} .
$$

For simplicity we define $Q_{p}^{(1)} \equiv Q_{p}$. Three types of UIRs are distinguished for dS group, $S O(1,4)$, according to the range of values of the parameters $q$ and $p[22,23]$, namely: the principal, complementary and discrete series. The flat limit indicates that in the principal and complementary series the value of $p$ bears meaning of the spin. For the discrete series the only representation which has a physically meaningful Minkowskian counterpart is $p=q$ case. Mathematical details of the group contraction and the physical principles underlying the relationship between $\mathrm{dS}$ and Poincaré groups can be found in Refs [24] and [25] respectively. We are interested in spin-2 representation since in the linear approximation graviton may be considered as a massless spin-2 field. The spin-2 tensor representations are classified with respect to the UIR of dS group as follows:

i) The UIRs $U^{2, \nu}$ in the principal series where $p=s=2$ and $q=\frac{1}{2}+i \nu$ correspond to the Casimir spectral values

$$
\left\langle Q_{2}^{\nu}\right\rangle=\nu^{2}-\frac{15}{4}, \quad \nu \in \mathbb{R},
$$

note that $U^{2, \nu}$ and $U^{2,-\nu}$ are equivalent.

ii) The UIRs $V^{2, q}$ in the complementary series where $p=s=2$ and $q-q^{2}=\mu$, correspond to

$$
\left\langle Q_{2}^{\mu}\right\rangle=q-q^{2}-4 \equiv \mu-4, \quad 0<\mu<\frac{1}{4} .
$$

iii) The UIRs $\Pi_{2, q}^{ \pm}$in the discrete series where $p=s=2$ correspond to

$$
\left\langle Q_{2}^{q}\right\rangle=-6-(q+1)(q-2), \quad q=1,2 .
$$


The "massless" spin-2 field in dS space corresponds to the $\Pi_{2,2}^{ \pm}$and $\Pi_{2,1}^{ \pm}$cases in which the sign \pm , stands for the helicity. In these cases, two representations $\Pi_{2,2}^{ \pm}$, in the discrete series with $p=q=2$, have a Minkowskian interpretation.

The compact subgroup of conformal group $S O(2,4)$ is $S O(2) \otimes S O(4)$. Let $C\left(E ; j_{1}, j_{2}\right)$ denote the irreducible projective representation of the conformal group, where $E$ is the eigenvalues of the conformal energy generator of $S O(2)$ and $\left(j_{1}, j_{2}\right)$ is the $\left(2 j_{1}+1\right)\left(2 j_{2}+1\right)$ dimensional representation of $S O(4)=S U(2) \otimes S U(2)$. The representation $\Pi_{2,2}^{+}$has a unique extension to a direct sum of two UIRs $C(3 ; 2,0)$ and $C(-3 ; 2,0)$ of the conformal group, with positive and negative energies respectively $[24,18]$. The latter restricts to the massless Poincaré $\operatorname{UIRs} P^{>}(0,2)$ and $P^{<}(0,2)$ with positive and negative energies respectively. $\overrightarrow{\mathcal{P}}^{<}(0,2)$ (resp. $\left.\overrightarrow{\mathcal{P}}^{<}(0,-2)\right)$ are the massless Poincaré UIRs with positive and negative energies and positive (resp. negative) helicity. The following diagrams illustrate these connections

$$
\begin{aligned}
& \mathcal{C}(3,2,0) \quad \mathcal{C}(3,2,0) \hookleftarrow \mathcal{P}^{>}(0,2) \\
& \Pi_{2,2}^{+} \hookrightarrow \begin{array}{c}
\oplus \\
\end{array}
\end{aligned}
$$

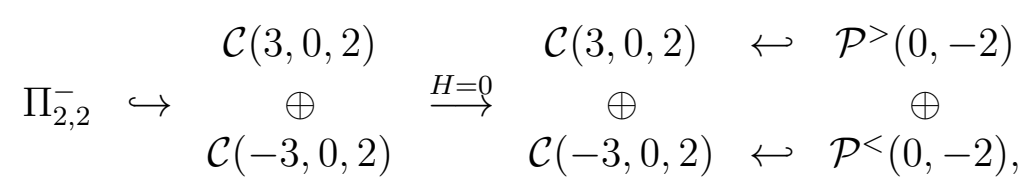

where the arrows $\hookrightarrow$ designate unique extension. It is important to note that the representations $\Pi_{2,1}^{ \pm}$do not have corresponding flat limit.

The action of the Casimir operators $Q_{0}$ and $Q_{2}$ on the symmetric rank-2 tensor field can be written in the more explicit form $[16]$

$$
Q_{2} \mathcal{K}_{\alpha \beta}=\left(Q_{0}-6\right) \mathcal{K}_{\alpha \beta}+4 x_{(\alpha} \bar{\partial} \cdot \mathcal{K}_{\beta)}-4 \bar{\partial}_{(\alpha} x \cdot \mathcal{K}_{\beta)}+2 \eta_{\alpha \beta} \mathcal{K}_{\alpha \beta}
$$

By imposing the conditions $\bar{\partial} \cdot \mathcal{K}=0=\mathcal{K}^{\prime}$ and $x \cdot \mathcal{K}=0, \mathcal{K}_{\alpha \beta}$ transforms according to the UIR of the dS group, so we have

$$
\begin{gathered}
Q_{2} \mathcal{K}_{\alpha \beta}=\left(Q_{0}-6\right) \mathcal{K}_{\alpha \beta}=<Q_{2}>\mathcal{K}_{\alpha \beta}, \\
Q_{2}=<Q_{2}>I_{d} \text { and } Q_{0}=\left(<Q_{2}>+6\right) I_{d}
\end{gathered}
$$

For the UIR of discrete series we have two possibilities, $<Q_{2}>=-6$ and -4 , which correspond to $\Pi_{2,2}^{ \pm}$and $\Pi_{2,1}^{ \pm}$, respectively; thus for these representations we have

$$
Q_{0} \mathcal{K}_{\alpha \beta}=0, \quad\left(Q_{0}-2\right) \mathcal{K}_{\alpha \beta}=0
$$

We consider two cases: $Q_{0} \mathcal{K}_{\alpha \beta} \neq 0$ and $\left(Q_{0}-2\right) \mathcal{K}_{\alpha \beta} \neq 0$. For the first case, we define the rank-2 tensor $A_{\alpha \beta}\left(\equiv Q_{0} \mathcal{K}_{\alpha \beta}\right)$; it will satisfy the similar conditions as $\mathcal{K}_{\alpha \beta}$, namely $\bar{\partial} \cdot A=0=A^{\prime}$ and $x \cdot A=0$. Now if $A_{\alpha \beta}$ obeys the following equation

$$
\left(Q_{0}-2\right) A_{\alpha \beta}=0 \text { or equivalently }\left(Q_{2}+4\right) A_{\alpha \beta}=0,
$$

it will transform according to the UIR of the dS group $\Pi_{2,1}^{ \pm}$.

And for the second one, we define the rank-2 tensor $B_{\alpha \beta}\left(\equiv\left(Q_{0}-2\right) \mathcal{K}_{\alpha \beta}\right)$ and similar statements hold for this case. Similarly, if $B_{\alpha \beta}$ obeys the following equation

$$
Q_{0} B_{\alpha \beta}=0 \text { or equivalently }\left(Q_{2}+6\right) B_{\alpha \beta}=0
$$


it will transform according to the UIR of the $\mathrm{dS}$ group $\Pi_{2,2}^{ \pm}$. Obviously in either cases $\mathcal{K}_{\alpha \beta}$ obeys the following equation as well

$$
Q_{0}\left(Q_{0}-2\right) \mathcal{K}_{\alpha \beta}=0, \quad \text { or }\left(Q_{2}+4\right)\left(Q_{2}+6\right) \mathcal{K}_{\alpha \beta}=0,
$$

note that $Q_{0}\left(Q_{0}-2\right)=\left(Q_{0}-2\right) Q_{0}$. Therefore the fields that satisfy Eq. (A.9) transform according to one of the UIR of the dS group namely, $\Pi_{2,1}^{ \pm}$or $\Pi_{2,2}^{ \pm}$.

The solutions of the Eq. (A.9) are important, Since they correspond to the UIRs of dS group. So the definition an homomorphism between $\mathcal{K}_{\alpha \beta}$ and a rank-3 mixed symmetry tensor field $F_{\alpha \beta \gamma}$ (Fierz representation), leads us to a gravitational field which transforms according to the UIR of conformal group as well [26]. In Ref. [20,27] we have considered the possible solutions for the Eq. (A.9) by taking $\mathcal{K}_{\alpha \beta}=\mathcal{D}_{\alpha \beta} \phi$, where $\mathcal{D}_{\alpha \beta}$ is a polarization tensor and $\phi$ is a scalar field in $\mathrm{d} S$ space.

Now we want to obtain the intrinsic counterpart of the Eq. (A.9), in other words we want to project it on the hyperboloid. From the definition of the Casimir operator in dS space, one can write $Q_{0}=-H^{-2} \bar{\partial}^{2}$. And also we have (Eqs. (3.12-13))

$$
\begin{gathered}
h_{\mu \nu}=\frac{\partial x^{\alpha}}{\partial X^{\mu}} \frac{\partial x^{\beta}}{\partial X^{\nu}} \mathcal{K}_{\alpha \beta}, \\
\square h_{\mu \nu}=H^{2} \frac{\partial x^{\alpha}}{\partial X^{\mu}} \frac{\partial x^{\beta}}{\partial X^{\nu}}\left(-Q_{0}-2\right) \mathcal{K}_{\alpha \beta}, \\
\square^{2} h_{\mu \nu}=H^{4} \frac{\partial x^{\alpha}}{\partial X^{\mu}} \frac{\partial x^{\beta}}{\partial X^{\nu}}\left(Q_{0}+2\right)\left(Q_{0}+2\right) \mathcal{K}_{\alpha \beta},
\end{gathered}
$$

note that we take $\bar{\partial} \cdot \mathcal{K}=0$ in $\mathcal{M}_{\gamma \alpha \beta}^{\gamma}$. By using the above relations, Eq. (A.9) in the intrinsic coordinate becomes:

$$
\left(\square^{2}+6 H^{2} \square+8 H^{4}\right) h_{\mu \nu}=0,
$$

it is clear this equation differs from Eq. (2.13) of the linear Weyl gravity.

\section{B Some useful mathematical relations}

The variation of the $W_{\mu \nu}^{(1)}$ and $W_{\mu \nu}^{(2)}$ contains some terms that are difficult to calculate especially in the curved background. The following expressions are important in deriving the variation of $W_{\mu \nu}[28]$

$$
\begin{gathered}
\delta \Gamma_{b d}^{a}=\frac{1}{2} g^{a e}\left(\nabla_{b} \delta g_{e d}+\nabla_{d} \delta g_{b e}-\nabla_{e} \delta g_{b d}\right) \\
\delta R_{b c d}^{a}=\frac{1}{2} g^{a e}\left(\nabla_{c} \nabla_{b} \delta g_{d e}+\nabla_{c} \nabla_{d} \delta g_{b e}-\nabla_{c} \nabla_{e} \delta g_{b d}-\nabla_{d} \nabla_{b} \delta g_{c e}-\nabla_{d} \nabla_{c} \delta g_{b e}+\nabla_{d} \nabla_{e} \delta g_{b c}\right) \\
\delta R_{a b}=\frac{1}{2} g^{c d}\left(\nabla_{c} \nabla_{a} \delta g_{b d}+\nabla_{c} \nabla_{b} \delta g_{a d}-\nabla_{c} \nabla_{d} \delta g_{a b}-\nabla_{b} \nabla_{a} \delta g_{c d}\right) \\
\delta R=g^{a b} g^{c d}\left(\nabla_{c} \nabla_{a} \delta g_{b d}-\nabla_{c} \nabla_{d} \delta g_{a b}\right)-R^{c d} \delta g_{c d} .
\end{gathered}
$$

Now the calculation of $R^{2}$ terms in the total action becomes straightforward

$$
\delta\left(\sqrt{-g} R^{2}\right)=2 \sqrt{-g} R \delta R+R^{2} \delta \sqrt{-g}=
$$




$$
\sqrt{-g} \nabla_{c} A^{c}+\sqrt{-g}\left(2 \nabla^{e} \nabla^{f} R-2 g^{e f} \square R-2 R R^{e f}+\frac{1}{3} g^{e f} R^{2}\right) \delta g_{e f},
$$

where

$$
A^{c} \equiv 2 g^{a b} g^{c d} R\left(\nabla_{a} \delta g_{b d}-\nabla_{d} \delta g_{a b}\right)-2 g^{c b} g^{a d} \nabla_{a} R \delta g_{b d}+2 g^{a b} g^{d c} \nabla_{d} R \delta g_{a b} .
$$

Similarly we can write

$$
\begin{gathered}
\delta\left(\sqrt{-g} R^{a b} R_{a b}\right)=\sqrt{-g} \nabla_{c} B^{c}+ \\
\sqrt{-g}\left(\nabla_{a} \nabla^{e} R^{a f}+\nabla_{a} \nabla^{f} R^{a e}-\square R^{e f}-g^{e f} \nabla_{a} \nabla_{b} R^{a b}-2 R_{a}^{f} R^{a e}+\frac{1}{2} g^{e f} R_{a b} R^{a b}\right) \delta g_{e f},
\end{gathered}
$$

where

$$
\begin{aligned}
B^{c}=g^{c d} R^{a b} & \left(\nabla_{a} \delta g_{b d}+\nabla_{b} \delta g_{a d}-\nabla_{d} \delta g_{a b}\right)-g^{a d}\left(\nabla_{a} R^{c b} \delta g_{b d}-\nabla_{b} R^{c d} \delta g_{a d}\right) \\
& -g^{b d}\left(R^{a c} \nabla_{a} \delta g_{b d}+\nabla_{b} R^{a c} \delta g_{a d}\right)+g^{d c} \nabla_{d} R^{a b} \delta g_{a b} .
\end{aligned}
$$

Note that the first terms in the right hand side of Eq.s(B.5-B.6) have no conurbation in considering of the equation of motion.

The linear form has been obtained by taking $\delta g_{a b}=h_{a b}$, and $\delta g^{a b}=-h^{a b}$. For example we obtain

$$
\begin{gathered}
\delta R_{a b}=-\frac{1}{2}\left(\square h_{a b}+\nabla_{b} \nabla_{a} h-\nabla_{c} \nabla_{b} h_{a}^{c}-\nabla_{c} \nabla_{a} h_{b}^{c}\right) \\
\delta R=-\square h+\nabla^{a} \nabla^{b} h_{a b}-R^{a b} h_{a b}, \\
2 \delta\left(\nabla_{c} \nabla_{d} R_{a b}\right)= \\
+\nabla_{b e}\left(\nabla_{c} \nabla_{d} h_{a}^{e}+\nabla_{c} \nabla_{a} h_{d}^{e}-\nabla_{c} \nabla^{e} h_{a d}\right)-R_{a e}\left(\nabla_{c} \nabla_{d} h_{b}^{e}+\nabla_{c} \nabla_{b} h_{d}^{e}-\nabla_{c} \nabla^{e} h_{b d}\right) \\
-\nabla_{c} R_{e b}\left(\nabla_{d} h_{a}^{e}+\nabla_{a} h_{d}^{e}-\nabla^{e} h_{a d}\right)-\nabla_{c} R_{e a}\left(\nabla_{d} h_{b}^{e}+\nabla_{b} h_{d}^{e}-\nabla^{e} h_{b d}\right) \\
-\nabla_{d} R_{e b}\left(\nabla_{c} h_{a}^{e}+\nabla_{a} h_{c}^{e}-\nabla^{e} h_{a c}\right)-\nabla_{d} R_{e a}\left(\nabla_{c} h_{b}^{e}+\nabla_{b} h_{c}^{e}-\nabla^{e} h_{b c}\right) \\
-\nabla_{e} R_{a b}\left(\nabla_{c} h_{d}^{e}+\nabla_{d} h_{c}^{e}-\nabla^{e} h_{d c}\right) .
\end{gathered}
$$

Multiplying the above equation by $g^{c a}$ and using the following relation

$$
g^{c a} \delta\left(\nabla_{c} \nabla_{d} R_{a b}\right)=\delta\left(\nabla_{c} \nabla_{d} R_{b}^{c}\right)+h^{c a} \delta\left(\nabla_{c} \nabla_{d} R_{a b}\right),
$$

one obtains the other form of variations, for example

$$
\begin{gathered}
2 \delta\left(\square R_{a b}\right)=-2 h^{d e} \nabla_{e} \nabla_{d} R_{a b}-\square\left(\square h_{a b}-\nabla_{b} \nabla_{a} h+\nabla_{c} \nabla_{b} h_{a}^{c}+\nabla_{c} \nabla_{a} h_{b}^{c}\right) \\
-R_{b c}\left(\square h_{a}^{c}+\nabla^{d} \nabla_{a} h_{d}^{c}-\nabla^{d} \nabla^{c} h_{a d}\right)-R_{a c}\left(\square h_{b}^{c}+\nabla^{d} \nabla_{b} h_{d}^{c}-\nabla^{d} \nabla^{c} h_{b d}\right)-2 \nabla^{d} R_{c b}\left(\nabla_{d} h_{a}^{c}+\nabla_{a} h_{d}^{c}\right. \\
\left.-\nabla^{c} h_{a d}\right)-2 \nabla^{d} R_{c a}\left(\nabla_{d} h_{b}^{c}+\nabla_{b} h_{d}^{c}-\nabla^{c} h_{b d}\right)-2 \nabla_{c} R_{a b} \nabla^{d} h_{d}^{c}+\nabla_{c} R_{a b} \nabla^{c} h, \\
\delta\left(\nabla_{a} \nabla_{b} R\right)=\nabla_{a} \nabla_{b}\left(-\square h+\nabla_{c} \nabla^{d} h_{d}^{c}\right)-\nabla_{a} \nabla_{b} h^{c d} R_{c d}-\nabla_{b} h^{c d} \nabla_{a} R_{c d} \\
-\nabla_{a} h^{c d} \nabla_{b} R_{c d}-\frac{1}{2}\left(\nabla_{a} h_{b}^{c} \nabla_{c} R+\nabla_{b} h_{a}^{c} \nabla_{c} R+\nabla^{c} h_{a b} \nabla_{c} R\right)-h^{c d} \nabla_{a} \nabla_{b} R_{c d},
\end{gathered}
$$




$$
\begin{gathered}
\delta(\square R)=-h^{a b} \square R_{a b}-h^{a b} \nabla_{b} \nabla_{a} R \\
-\square^{2} h+\square \nabla_{b} \nabla^{a} h_{a}^{b}-\square h_{a b} R^{a b}-2 \nabla^{c} h^{a b} \nabla_{c} R_{a b}-\nabla^{c} h_{c}^{b} \nabla_{b} R+\frac{1}{2} \nabla^{c} h \nabla_{c} R .
\end{gathered}
$$

Now one can easily rewrite these variations in the dS background noting that de Sitter space is a maximally symmetric space so Riemannian tensor takes the following form

$$
R_{a b c d}=H^{2}\left(g_{a c} g_{b d}-g_{a d} g_{b c}\right)
$$

Ricci tensor and Ricci scalar become

$$
R_{b d}=3 H^{2} g_{b d}, \text { and } R=12 H^{2} .
$$

On the other hand we have

$$
\left[\nabla_{c}, \nabla_{f}\right] h_{b}^{a}=R_{d c f}^{a} h_{b}^{d}-R_{b c f}^{d} h_{d}^{a},
$$

this relation in $\mathrm{dS}$ background becomes

$$
\left[\nabla_{c}, \nabla_{f}\right] h_{b}^{a}=H^{2}\left(g_{c}^{a} h_{f b}-g_{f}^{a} h_{c b}-g_{b f} h_{c}^{a}+g_{b c} h_{f}^{a}\right)
$$

\section{References}

[1] V. Faraoni, E. Gunzing and P. Nardone " conformal transformations in classical gravitational theories and in cosmology", Fund. Cosmicphys. 20 (1999) 121. arXiv: gr-qc/9811047.

[2] C. Fronsdal, Phys. Rev. D 30 (1984) 2081.

[3] P.D. Mannheim, Astrophys. J. 479 (1997) 659.

[4] S. Capozziello, et.al., arXiv: gr-qc:07122980.

[5] B.S. DeWitt, Phys. Rep., 19C (1975) 295; K.S. Stelle, Gen. Rel. and Grav. 9 No. 4 (1978) 353.

[6] P.D. Mannheim and D. Kazanas Phys. Rev. D 44 (1991)417; P.D. Mannheim, Astrophys. J. 391 (1992) 429; Astrophys. J. 419 (1993)150; Phys. Rev. D. 58 (1998) 103511.

[7] P.D. Mannheim, Gen. Relativ. Gravi. 22 (1990) 289.

[8] P.D. Mannheim and D. Kazanas, Astrophys. J. 342 (1989) 635.

[9] P.D. Mannheim, Conformal Gravity Challenges String Theory, arXiv: 0707.2283v1.

[10] P.D. Mannheim, Phys. Rev. D. 75 (2007) 124006.

[11] P. Chen arXiv: gr-qc 1002.4275; A. Bhattacharya and et. al., arXiv: gr-qc 0910.1112.

[12] C.W. Misner, K.S. Thorne and J.A. Wheeler Gravitation, New York, (1973); R.M. Wald, General Relativity, Chicago University press, (1984). 
[13] B. S. DeWitt, Relativity, Groups and Topology, C. DeWitt and B. S. DeWitt, Eds., Gordon and Breach New York (1964).

[14] T. Garidi, J. P. Gazeau, S. Rouhani, and M.V. Takook, J. Math. Phys. 49 (2008) 032501.

[15] P.A.M. Dirac, Annals of Math. 37 (1935-b) 429.

[16] T. Garidi, J. P. Gazeau and M.V. Takook, J. Math. Phys. 44 (2003) 3838.

[17] J. Bouchami, M.B. Paranjape, Phys. Rev. D 78 (2008) 044022; L. Fabbri, M. B. Paranjape, Zero-energy plane waves in conformal gravity, arXiv: 0812.2491.

[18] A. O. Barut and A. Böhm, J. Math. Phys. 11 (1970) 2938.

[19] B. Binegar, C. Fronsdal, and W. Heidenreich, Phys. Rev. D 27(1983)2249.

[20] M. Dehghani, S. Rouhani, M.V. Takook and M.R. Tanhayi, Phys. Rev. D 77 (2008) 064028.

[21] C. Fronsdal and W.F. Heidenreich, J. Math. Phys. 28 (1987) 215.

[22] J. Dixmier, Bull. Soc. Math. France 89 (1961) 9.

[23] B. Takahashi, Bull. Soc. Math. France 91 (1963) 289.

[24] M. Levy-Nahas, J. Math. Phys. 8 (1967) 1211.

[25] H. Bacry, J.M. Levy-Leblond, J. Math. Phys. 9 (1968) 1605.

[26] M.V. Takook, M.R. Tanhayi and S. Fatemi, J. Math. Phys. 51 (2010) 032503. arXiv:0903.5249v1.

[27] H. Pejhan, M.R. Tanhayi and M.V. Takook, Int. J. Theor. Phys. 49 (2010) 2263.

[28] N.H. Barth and S.M. Christensen, Phys. Rev. D 28 (1983) 1876. 